\title{
Characterisation of the dysferlin skeletal muscle promoter
}

\author{
RM Foxton ${ }^{1}$, SH Laval ${ }^{1}$ and KMD Bushby*,1 \\ ${ }^{1}$ Institute of Human Genetics, International Centre for Life, Central Parkway, Newcastle-upon-Tyne NE1 3BZ, UK
}

Deficiency of the skeletal muscle membrane protein dysferlin causes the related and overlapping neuromuscular disorders limb-girdle muscular dystrophy type 2B (LGMD2B) and Miyoshi myopathy. This paper describes the preliminary characterisation of the human dysferlin promoter. The transcriptional start site of dysferlin has been mapped using $5^{\prime}$ RACE PCR, which extended the length of the known $5^{\prime}$ UTR to $914 \mathrm{bp}$. Promoter elements have been mapped by assessing the ability of fragments from this region to activate the expression of a luciferase reporter gene borne on a plasmid transfected into differentiated and undifferentiated $\mathrm{C} 2 \mathrm{C} 12$ mouse myoblast cells. Finally, the core promoter region has been screened for mutations in suspected dysferlinopathy patients.

European Journal of Human Genetics (2004) 12, 127-131. doi:10.1038/sj.ejhg.5201092

Published online 22 October 2003

Keywords: dysferlin; muscular dystrophy; promoter

\section{Introduction}

Dysferlin is a large $(230 \mathrm{kDa})$ skeletal muscle protein which is deficient in the related conditions limb-girdle muscular dystrophy type $2 \mathrm{~B}$ and Myoshi myopathy (MM). ${ }^{1,2}$ Dysferlin expression is detectable very early during the specification of the skeletal muscle and is strongly expressed in the adult skeletal muscle, where it localises to the sarcolem$\mathrm{ma},{ }^{3}$ although it is not part of the dystrophin-glycoprotein complex. ${ }^{4}$ Dysferlin has been shown to associate with caveolin-3 by coimmunoprecipitation, ${ }^{5}$ and is detected intracellularly in a number of limb-girdle muscular dystrophies. ${ }^{6}$ However, the function of this protein remains unresolved.

The characterisation of the $5^{\prime}$ UTR and promoter region of the dysferlin gene using $5^{\prime} \mathrm{RACE}$, and luciferase reporter gene activity of constructs containing these sequences in C2C12 myoblast cells, is presented here. We show that the regulation of dysferlin transcription is complex with the predicted involvement of both positive and negative regulatory elements.

*Correspondence: Dr KMD Bushby. Tel: + 44191241 8737; Fax: + 44191-241-8666; E-mail: kate.bushby@ncl.ac.uk

Received 3 April 2003; revised 18 June 2003; accepted 18 July 2003
Materials and methods

5' RACE

5' RACE was carried out on human skeletal muscle marathon-ready cDNA (Clontech) using 5'-tcatcttccaccccegccgcccttcc- $3^{\prime}$ and $5^{\prime}$-ccggctgcggagtctcccaatgacacg$3^{\prime}$ (positions 109 to 84 and 54 to 28 of Accession AF075575 and positions +650 to +625 and +595 to +569 relative to the transcriptional start site) as primary and secondary dysferlin-specific primers. PCR products were cloned into pGEM-T Easy (Promega) and then sequenced using standard techniques. The sequences were analysed for transcription factor binding sites using the programmes MatInspector V2.2 (http://transfac.gbf.de/TRANSFAC) and TESS (Transcription Element Search System; http:// www.cbil.upenn.edu/tess).

\section{Plasmid constructs}

A 3263 bp NheI-HindIII fragment of cosmid clone ae43f21 (LLNL Chr2 Cosmid library) containing nucleotides -2035 to +1222 relative to the transcriptional start site was cloned into the pGL3-Basic vector (Promega) upstream of the firefly luciferase reporter gene. Further subclones constructed in the same vector using standard molecular 
biology techniques contained the following restriction fragments (see Figure 3a for details): NheI-BamHI (-2035 to +338$)$; BamHI-HindIII $(+338$ to +1222$)$; NsiI-HindIII $(-875$ to +1222$)$; NheI-NsiI (-2035 to -875$)$; NsiI-BamHI $(-875$ to +338$)$; HincII-HindIII $(-265$ to +1222$)$; NheIHincII (-2035 to -265$)$; NsiI-HincII $(-875$ to -265$)$. In addition, the entire NheI-HindIII fragment was cloned into pGL3-Basic in the opposite orientation (antisense construct).

\section{Culture of $\mathrm{C} 2 \mathrm{C} 12$ mouse myoblasts}

C2C12 mouse skeletal muscle myoblasts (ECACC) were cultivated in DMEM containing 15\% foetal bovine serum supplemented with $2 \%$ chick embryo extract or DMEM containing 3\% horse serum for differentiation (Gibco-Invitrogen). For each experiment, three wells of $30-50 \%$ confluent C2C12 cells were cotransfected with a mixture of $500 \mathrm{ng}$ of the experimental plasmid and $50 \mathrm{ng}$ of pRL-SV40 (Promega) internal control plasmid, which expresses the Renilla luciferase reporter gene using Fugene 6 Transfection Reagent (Roche) in growth medium according to the manufacturer's protocol. Following a day of growth, the cells were either harvested for the day 0 time point or the medium changed either for fresh growth medium in the case of undifferentiated cells or differentiation medium.

\section{Western blotting}

Cell pellets derived from $\mathrm{C} 2 \mathrm{C} 12$ cultures at various stages of differentiation were resuspended directly in PAGE buffer and separated on $4-12 \%$ bis-tris polyacrylamide gels (NOVEX NuPage from Invitrogen) using NuPage MES buffer system (Invitrogen). Western blots were prepared using Hybond-C extra (Amersham), and stained for dysferlin using NCL-Hamlet (the kind gift of Dr Louise Anderson, University of Newcastle) and GS-28 (BD-Transduction laboratories) using standard techniques.

\section{Luciferase reporter gene assay}

Firefly and Renilla reporter genes were sequentially assayed using the Dual-Luciferase Reporter Assay System Kit (Promega) using a Microlumat plus automated luminometer (Perkin-Elmer Instruments). A volume of $10 \mu \mathrm{l}$ of the thawed cell lysates was combined with $50 \mu \mathrm{l}$ of Luciferase Assay Reagent II, followed by $50 \mu$ l of Stop and Glo Reagent. All readings were taken in triplicate.

\section{Mutation analysis}

The dysferlin skeletal muscle promoter region, as defined above, was sequenced in 14 patients with muscular dystrophy, in whom mutations in the coding sequence of the gene had been excluded by DHPLC. These patients were suspected to have LGMD/MM either clinically, by linkage to the dysferlin gene, or by the finding of a reduction in dysferlin on protein analysis.

\section{Results}

Sequence analysis of the $5^{\prime}$ RACE products extended the known dysferlin 5' UTR (Accession AF075575) by 545 bp, bringing the total length of the $5^{\prime}$ UTR to 914 bp upstream of the reported translational start site. A minority of RACE products terminated after $423 \mathrm{bp}$ of $5^{\prime}$ UTR sequence, representing a potential alternative transcriptional start site. Alternatively, these may represent premature termination of the RACE products caused by secondary structure of the messenger RNA or other artefacts of library construction. The genomic sequence (2939 bp; Accession \#AJ566204) upstream of the $5^{\prime}$ RACE product was obtained from clone ae43f21 (LLNL Chr2 Cosmid library), representing $3853 \mathrm{bp}$ upstream of exon 1 , which is in complete concordance with the subsequently determined sequence of BAC clone RP11-601I8 (15324-19176 of Accession \#AC104084; also containing the first 16 exons of dysferlin) with the exception of three single base pair substitutions which probably represent polymorphic sites. A 669 bp CpG island was predicted by NIX (http://www.hgmp.mrc.ac.uk/ NIX/) and extends from +438 to 105 bp into intron 1 (see Figure 3b).

An initial time course of dysferlin protein expression in C2C12 myoblasts demonstrated that this cell line displayed some expression even in an undifferentiated state, and the protein expression was highly induced upon differentiation (Figure 1). Therefore, various constructs containing the human dysferlin $5^{\prime}$ flanking sequence were

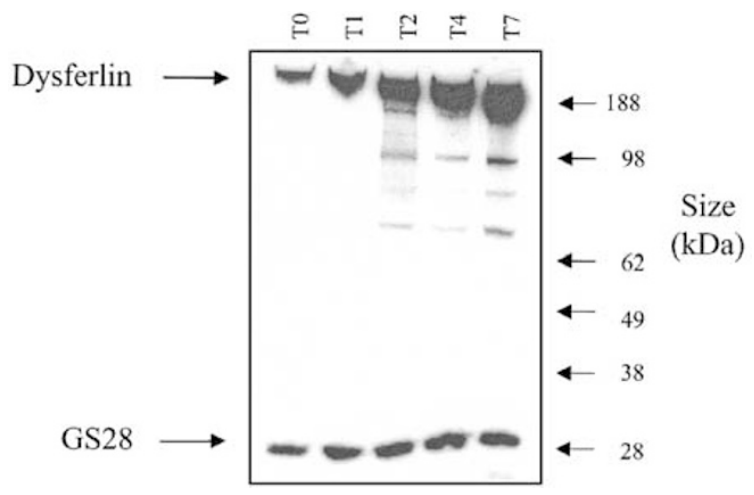

Figure 1 Expression of dysferlin in C2C12 myoblasts during differentiation. Western blot of cell lysates from C2C12 myoblasts prior to differentiation (T0) or following $1,2,4$ and 7 days of differentiation (T1, T2, T4 and T7, respectively) stained for dysferlin and GS-28 (a golgispecific marker) as a control protein. 
tested for their ability to direct reporter gene activity in undifferentiated and differentiated $\mathrm{C} 2 \mathrm{C} 12$ cells.

An NheI-HindIII fragment containing $2037 \mathrm{bp}$ of $5^{\prime}$ flanking sequence, exon 1 (1002 bp) and $224 \mathrm{bp}$ of intron 1 , including the entire $\mathrm{CpG}$ island $(-2035$ to +1222 relative to the transcriptional start site), displayed promoter activity as indicated by the induction of luciferase reporter gene expression in $\mathrm{C} 2 \mathrm{C} 12$ cells (Figure 2a).

In order to further define the sequences responsible for this activity, subfragments obtained from the NheI-HindIII sequence were also assayed for induction of luciferase reporter gene expression. Figure $2 \mathrm{~b}$ shows that an NheIBamHI (-2035 to +338) fragment absent for the CpG island also contains promoter activity, but reduced relative to the NheI-HindIII fragment $(-2035$ to +1222$)$. In contrast, the complementary construct containing the BamHI-HindIII fragment $(+338$ to +1222$)$ contained no significant promoter activity (data not shown).

The NsiI-HindIII fragment $(-875$ to +1222$)$, which contains the putative $\mathrm{CpG}$ island sequence also demonstrated significant promoter activity in this assay (Figure 2c), although reduced relative to the NheI-HindIII fragment. The residual NheI-NsiI fragment (-2035 to 875) displayed no significant promoter activity in these assays (data not shown).

The NsiI-BamHI fragment $(-875$ to +338$)$, which is common to both the NheI-BamHI and the NsiI-HindIII fragments, was then assayed for promoter activity. While this fragment contains the majority of the transcriptional activation which had been observed in the larger constructs at day 5 of differentiation (Figure $2 \mathrm{~d}$ ), the promoter

Figure 2 Mapping of the dysferlin transcriptional regulatory regions. A diagram of each fragment tested is shown on the left, with the results shown in the bar charts on the right. C2C12 cells were harvested prior to differentiation (day 0), or harvested on days 1, 2 and 5 with or without differentiation into myotubes. Reporter gene expression, as measured by firefly luciferase activity in the lysates of the experimental constructs or negative control (empty pGL3Basic vector), was normalised for transfection efficiency using the expression of Renilla luciferase from the pRL-SV40 internal control, and is therefore expressed in relative light units. Shaded bars show the average reporter gene activity from triplicate measurements of the (a) Nhel-Hindll construct, (b) Nhel-BamHI construct, (c) Nsil-HindIII construct, (d) Nsil-BamHI construct, (e) Nhel-Hincll construct, (f) Nsil-Hincll construct and (g) Hindlll-Nhel anti-sense construct. Open bars represent the background as they show the results for the negative control. Error bars on each reading correspond to the standard error. The relative light units are shown on the same scale on all the charts except those for the Nsil-Hincll and Nhel-Hincll constructs. On all the charts, the vertical lines are positioned every 0.02 relative light units.

activity observed at other time points and in undifferentiated cells was significantly reduced.

The promoter region sequence around the HincII site was further analysed. The HincII-HindIII fragment (-265 to +1222 ) showed no significant promoter activity (data not
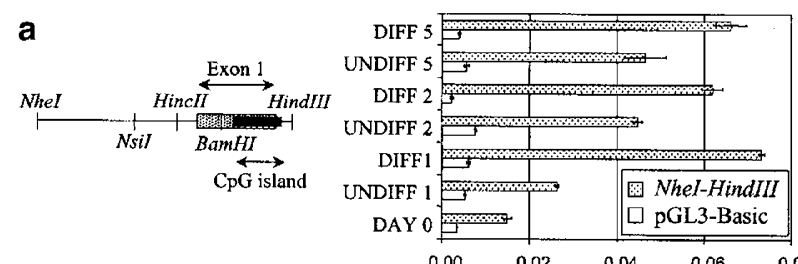

b
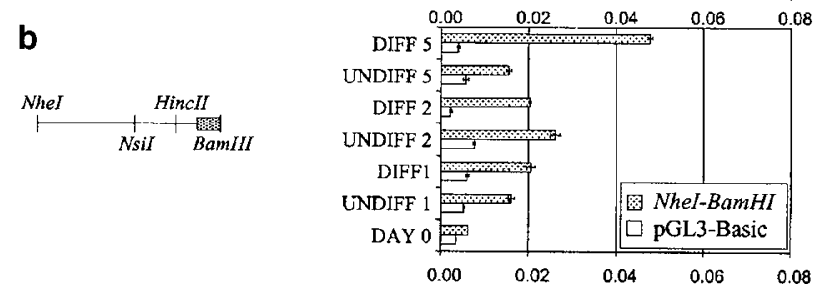

c

d

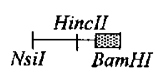

\section{e}

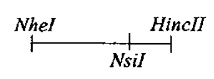

f

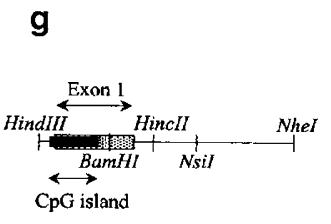

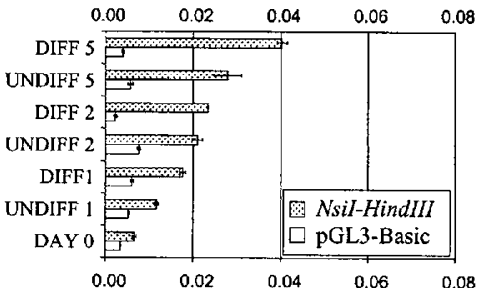
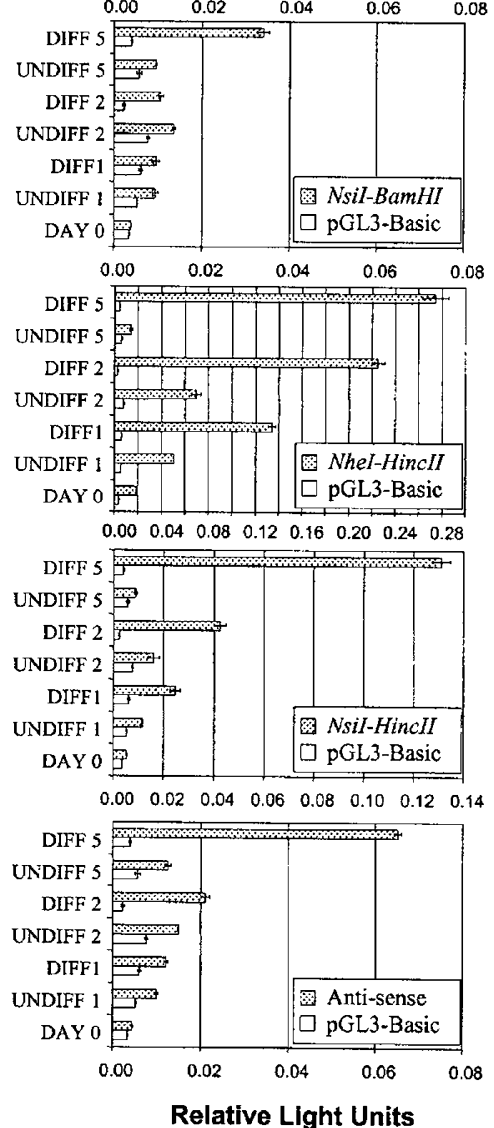
shown). However, the complementary NheI-HincII fragment ( -2035 to -265$)$ directed reporter gene expression more potently than any other construct tested (Figure 2e), including the original NheI-HindIII fragment, indicating that there is a strong negative regulatory element located between the HincII and BamHI sites. This was confirmed by contrasting the very high activity of the $610 \mathrm{bp} \mathrm{NsiI-}$ HincII fragment (-875 to -265 ; Figure 2f) with that of the $1217 \mathrm{bp} \mathrm{Nsi-BamHI}$ fragment $(-875$ to +338 ; Figure 2d).

Finally, we have demonstrated that this region of the genome also directs transcriptional activity, particularly in differentiated skeletal muscle cells, in the opposite direction from dysferlin transcription by cloning the NheIHindIII fragment in the antisense orientation into pGL3Basic (Figure 2g).

The sequence was analysed for the presence of skeletal muscle-specific mammalian transcription factors or widely expressed factors common in the promoters of skeletal muscle genes. There are predicted transcription factor binding sites for MyoD, Myogenin, TEF-1 and Sp1 between the NsiI and HincII sites. Between the NheI and NsiI sites, there are sites for MEF-2, MyoD, Myogenin and Sp1 (most present at the $5^{\prime}$ end of the region). Between BamHI and
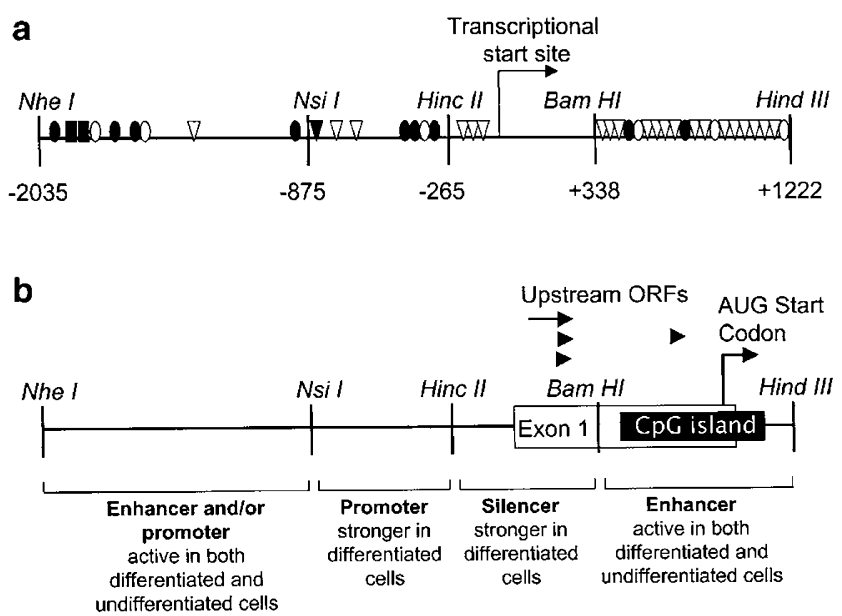

Figure 3 The elements involved in dysferlin gene expression. (a) The Nhel-HindlII fragment analysed for dysferlin promoter activity indicating the position (relative to the transcriptional start site) of the restriction sites used in luciferase constructs and the approximate position of consensus binding sites for the following transcription factors: TEF1 ( $\nabla), \operatorname{Sp1}(\nabla)$, MyoD ( $(\bullet)$, myogenin $(\bigcirc)$ and MEF-2 ( $\square$ ). (b) The same region of the dysferlin upstream sequence indicating the position of exon 1, intron 1, the predicted $\mathrm{CpG}$ island, translational start codon and the putative regulatory elements of the dysferlin promoter. Arrows above exon 1 represent upstream open reading frames, which may be involved in the regulation of dysferlin translation.
HindIII, there are sites for MyoD, Myogenin and Sp1. These are represented in Figure 3a.

Comparative analysis with the equivalent region of the mouse dysferlin gene (Accession \#AJ566205) showed no detectable sequence homology and no conservation of the pattern of transcription factor binding sites (data not shown).

Sequence analysis of the core dysferlin promoter fragment (ie the region defined by the NsiI-BamHI sites described above) in 14 suspected dysferlinopathy patients, for whom no mutations have been defined despite extensive screening of the coding region of dysferlin, revealed no sequence alterations.

\section{Discussion}

The complex regulation of dysferlin expression is probably effected by numerous sequence elements, some of which have been provisionally identified in this paper. The relatively long 5' UTR (914 bp) contains 13 upstream AUG start codons, four of which lie within a consensus sequence for initiation of translation. All the four open reading frames terminate within the $5^{\prime}$ UTR preceding the dysferlin translational start site. These may be translated in vivo, producing peptides of 74, 15, 4 and 12 residues. Studies of other genes have shown that upstream open reading frames can be involved in the regulation of expression by modulating the efficiency of translational initiation. $^{7}$

Analysis of the reporter gene construct data allows a model of dysferlin transcriptional regulatory regions to be proposed (Figure $3 \mathrm{~b}$ ). Comparing the results from Figure $2 \mathrm{a}-\mathrm{d}$ demonstrates the presence of positive regulatory elements flanking the core NsiI-BamHI fragment, which are especially required for activity in undifferentiated cells. The complexity of dysferlin transcriptional regulation is underlined by the presence of a silencer element between the HincII and BamHI restriction sites, as demonstrated by comparing the relative light unit values in Figure 2e with a and Figure $2 \mathrm{f}$ with d. However, the possibility that this silencer is an artefact caused by inclusion of the dysferlin transcriptional start site and exon 1 splice donor site in certain constructs cannot be excluded. Although the results of the luciferase reporter gene studies are consistent with the protein expression data, the magnitude of the induction of dysferlin expression in vivo is not replicated in the transfection studies. This may be due to the presence of additional regulatory elements in vivo that we have not included in the plasmid constructs, or to the loss of plasmid DNA during differentiation of the C2C12 myotubes, which would attenuate the expression profile over time.

Characterisation of the dysferlin promoter will allow any link between its transcriptional regulation and the primary protein defect to be identified, thereby contributing to the 
understanding of the pathological mechanisms involved in muscular dystrophy.

The experience of our laboratory is that around $50 \%$ of suspected dysferlinopathy patients ascertained in a number of ways, including clinically, by linkage to chromosome 2 p13 and by the finding of a reduction of dysferlin in skeletal muscle on immunoanalysis, have no detectable mutations in the coding sequence of the gene. However, there is only a small minority of patients in whom just a single mutant allele is detected. Various alternative explanations are possible to explain these findings - the Miyoshi myopathy phenotype is known to be genetically heterogeneous; ${ }^{8}$ so for the patients clinically ascertained, the mutation may lie in another gene altogether. This is less likely for the families in which linkage to chromosome 2 p13 has been established. For the patients with a reduction in dysferlin on immunolabelling of skeletal muscle biopsies, an alternative explanation is that these patients have mutations in an as yet undescribed muscular dystrophy gene, which cause a secondary reduction in dysferlin expression. Previous studies using immunofluorescence have shown that dysferlin may be secondarily downregulated in a number of muscular dystrophies. ${ }^{6}$ Alternatively, these patients may have mutations at the dysferlin locus, which are not detectable with the technologies currently used. This would include large deletions of the coding sequence, although these are not known to be prevalent in this disease, and mutations of the promoter. We tested the hypothesis that some patients might have mutations in the dysferlin promoter; however, we have sequenced the majority of the region described above in 14 such patients and found no significant alterations.

\section{Acknowledgements}

This work was supported by Action Research and the Muscular Dystrophy Campaign

\section{References}

1 Bashir R, Britton S, Strachan $\mathrm{T}$ et al.: A gene related to Caenorhabditis elegans spermatogenesis factor fer-1 is mutated in limb-girdle muscular dystrophy type 2B. Nat Genet 1998; 20: $37-42$.

2 Liu J, Aoki M, Illa I et al.: Dysferlin, a novel skeletal muscle gene, is mutated in Miyoshi myopathy and limb girdle muscular dystrophy. Nat Genet 1998; 20: 31-36.

3 Anderson LV, Davison K, Moss JA et al.: Dysferlin is a plasma membrane protein and is expressed early in human development. Hum Mol Genet 1999; 8: 855-861.

4 Matsuda C, Aoki M, Hayashi YK, Arahata K, Brown RH: Dysferlin is a surface membrane-associated protein that is absent in Miyoshi myopathy. Neurology 1999; 53: 1119-1122.

5 Matsuda C, Hayashi YK, Ogawa M et al.: The sarcolemmal proteins dysferlin and caveolin-3 interact in skeletal muscle. Hum Mol Genet 2001; 10: 1761-1766.

6 Piccolo F, Moore S A, Ford GC, Campbell KP: Intracellular accumulation and reduced sarcolemmal expression of dysferlin in limb-girdle muscular dystrophies. Ann Neurol 2000; 48: 902-912.

7 Kozak M: Constraints on reinitiation of translation in mammals. Nucleic Acids Res 2001; 29: 5226-5232.

8 Linssen WHJP, de Visser M, Notermans NC et al.: Genetic heterogeneity in Miyoshi-type distal muscular dystrophy. Neuromusc Disord 1998; 8: 317-320. 